\title{
Individual behavior and emergent properties of fish schools: a comparison of observation and theory
}

\author{
Steven V. Viscido ${ }^{1,2, *}$, Julia K. Parrish ${ }^{1,2}$, Daniel Grünbaum ${ }^{3}$ \\ ${ }^{1}$ Department of Biology, ${ }^{2}$ School of Aquatic and Fishery Sciences, and ${ }^{3}$ School of Oceanography, Box 351800 Kincaid Hall, \\ University of Washington, Seattle, Washington 98195, USA
}

\begin{abstract}
Polarity, group velocity, and inter-individual spacing are characteristics of fish schools that strongly affect individual school members. However, these characteristics are group-level 'emergent properties': collective outcomes of behavioral interactions among members, not under direct control of any single member. The relationships between members' behaviors and the emergent group properties they produce are complex and poorly understood. In this study, we quantified 3D trajectories of all individual fish within 4- and 8-fish populations of Danio aequipinnatus, using stereo videography and a computerized tracking algorithm. We compared group polarity, group speed, and mean nearest-neighbor distances of schools within these populations to a simulation model that explored how fish responded to attraction/repulsion, alignment and random forces. Real fish exhibited a high degree of temporal variability in both polarity and group speed. Polarity and speed of simulated schools depended very strongly on the strength of the alignment force. Time-averaged polarity of real fish schools was most similar to simulated schools when alignment force was 1 to $5 \%$ of the attraction/repulsion force. For both real and simulated fish, a clear relationship existed between group speed and polarity: polarized groups were faster than non-polarized groups. We propose a multi-dimensional state space where several emergent property statistics are represented along the axes, and suggest certain 'preferred' ranges of state space within which animal groups tend to localize, and in which they can sustain distinct types of regular architecture.
\end{abstract}

KEY WORDS: Social aggregation · Schooling behavior · Emergent properties · Polarity · Group speed $\cdot$ Nearest-neighbor distance $\cdot$ Danio aequipinnatus

\section{INTRODUCTION}

Group formation is prevalent amongst almost all animal taxa (Wilson 1975). For example, more than 50\% of fish species form schools (Shaw 1978), and $50 \%$ of bird species form feeding flocks (Lack 1968). Grouplevel characteristics - such as regular inter-individual spacing, a particular degree of polarization, or a characteristic group velocity-are generally believed to have important biological consequences (Parrish \& Edelstein-Keshet 1999), potentially affecting member fitness by (1) determining foraging success (Cody 1971, Krebs et al. 1972), (2) providing defense against, or escape from, predation (Hamilton 1971, Vine 1971, Watt et al. 1997, Viscido \& Wethey 2002), and (3) im- proving reproductive success (Lack 1968, Burger \& Gochfeld 1991). However, because they result from collective interactions and are not under direct control of any group member, these characteristics are not under simple, direct selection. Instead, selection on schooling behaviors reflects the complex dynamics of social interactions within groups: the fitness of a novel schooling behavior is mediated partly through its effects on others in the group, as well as the changes in group characteristics that consequently result. Thus, traits with short-term benefits to individual members may fail to arise because of overriding negative longterm consequences at the group level. Conversely, traits that are beneficial at the group level may fail to persist because individuals who do not exhibit them 
enjoy a relative advantage. Furthermore, some desirable group traits may be unattainable simply because no individual behaviors exist that could generate them.

Group characteristics that result from decentralized interactions are termed 'emergent properties' (Clark et al. 1997). Emergent properties of fish schools and bird flocks in particular have been frequently studied using simulation models. In most such models, each individual's behavioral choices are interpreted as a set of forces that affect the velocity or heading of an individual (e.g. Okubo 1986, Huth \& Wissel 1990, Flierl et al. 1999). These forces include biomechanical and environmental forces such as drag (Flierl et al. 1999), attraction and repulsion forces between sets of neighbors (Warburton \& Lazarus 1991, Romey 1996, Couzin et al. 2002), alignment or behavior-matching forces (Aoki 1982, Huth \& Wissel 1992), and randomness (Reuter \& Breckling 1994, Vabo \& Nottestad 1997, Stocker 1999). Simulation models typically connect specific individual behaviors to emergent properties by following each individual's position over time and statistically quantifying group-level characteristics.

The strength of the modeling approach is the relative ease of exploring many behavioral variants; however, there are also weaknesses. Chief among these are (1) that it is unclear which statistical measures best reflect biologically important characteristics and (2) that few quantitative observations of fish movements inside schools are detailed and long enough to provide a basis for comparison with model assumptions (Partridge \& Pitcher 1980, Aoki 1984, Parrish \& Turchin 1997, Hiramatsu et al. 2000). The lack of observational data is primarily due to the difficulties of tracking relatively large, fast objects (e.g. fish, birds) in 3D space over an extended period of time (Parrish et al. 2002). These observational difficulties are an important limitation on our understanding of how social animal groups function and how the underlying social behaviors evolved.

In this study, we develop a quantitative database of individual movements within fish schools and compare it with a simplified individual-based model of fish schooling using a specific subset of emergent property statistics - mean nearest-neighbor distance (NND), group speed, and polarity-applied analogously to both real and model fish trajectories. We assess which parameter values most closely correspond to actual fish behaviors, and which of our group-level statistics appear most informative about the biological functions of fish schools. Specifically, because one of the more striking emergent properties of fish schools is their polarized arrangement (Couzin et al. 2002, Parrish et al. 2002), we focus our simulations on one behavioral force, the alignment force, and compare the emergent properties observed in simulations with those of real fish schools in laboratory populations by systematically varying that force.

We present here the observational methods we used to quantify fish movements, followed first by descriptions of our schooling simulations and then by summaries of the emergent property statistics. We show how these statistics differed between our observations and simulations, and discuss the implications of our results for the mechanisms of social group formation. Finally, we suggest future directions in the analysis of these mechanisms.

\section{MATERIALS AND METHODS}

Biological observations. We observed 4- and 8-fish groups of giant danios Danio aequipinnatus. This species was also the physical model for our simulated fish. The danios were $5.3 \mathrm{~cm}$ long on average $( \pm 0.6 \mathrm{SD}, \mathrm{N}=$ 14 fish measured) and had a mass of $1.7 \mathrm{~g}( \pm 0.5 \mathrm{SD}$, $\mathrm{N}=14$ fish measured). Danios were held in two $600 \mathrm{l}$ holding tanks on a fixed 14:10 h light:dark cycle and fed ad libitum. At 09:00 h on the day of an observation, 4 or 8 fish were haphazardly selected from holding tanks and placed into a $1 \mathrm{~m}^{3}$ clear acrylic observation tank. The observation tank contained still water at the same temperature as the holding system, and was illuminated by nine $100 \mathrm{~W}$ floodlights equipped with fresnel diffusers and arranged to minimize both glare and shadows. Fish were filmed against a white background. Pilot trials showed no difference in behavior among acclimation times in the observation tank ranging from 6 to $24 \mathrm{~h}$. Therefore, we acclimated animals for $6 \mathrm{~h}$ before recording their behavior.

We used Panasonic PVDV-401 mini-DV video cameras mounted directly over the tank (to record $x-y$ movements) and at floor level (to record $x-Z$ movements) connected by firewire to 2 Sony DSR-20 digital tape decks. Tape decks and monitors were housed behind a screen such that an observer could operate the decks and observe fish behavior in real time without disturbing the animals. After filming for $30 \mathrm{~min}-$ utes, the fish were removed from the observation tank, and a metal calibration frame was inserted. The frame consisted of a $7 \times 7$ square grid of small black beads located $14 \mathrm{~cm}$ apart. The frame was filmed in 4 different positions, facing toward and away from each camera to establish a coordinate system just inside the outer edges of the tank.

Preliminary trials indicated that fish in small groups occasionally spend time nudging against the sides of the tank, interacting with their own reflections rather than with other fish ('glass kissing'). Because glass kissing is clearly an artifact of the observational conditions and not a part of the animals' natural behavior, 
only video sequences with more than 10 consecutive minutes of 'natural' swimming within the central volume of the tank were retained. We then randomly selected a 5 min subset of the natural schooling behavior in each trial for analysis. Below, we present results from three 4 -fish trials and three 8 -fish trials.

Image capture and conversion: Digital recordings of fish observations were transferred via firewire to an Apple G4 Power PC computer at the standard NTSC frame rate $(29.97 \mathrm{~Hz})$ and converted to QuickTime movie format using Adobe Premier 5.1 for Macintosh. We divided each $5 \mathrm{~min}$ digital movie into smaller 30 s (900 frame) segments in Adobe Premier to facilitate analysis. Pixel coordinates of fish centroids in each camera view were obtained using automated $\mathrm{NIH}$ Image (v. 1.62 for Macintosh) macros. Analysis steps included background subtraction using the timeaveraged image, thresholding, and recording of positions to an ASCII text file.

Trajectory reconstruction: The program we used for trajectory reconstruction, Tracker3D, was written inhouse using MatLab (The Mathworks 2001, Release 12.1). This program used pixel coordinate data and calibration images to convert each fish position in each video frame to a ray through space in a real-world coordinate system, and then reconstructed fish trajectories within a camera view by associating the corresponding fish centroids in sequential frames using nearest-neighbor criteria. This simple criterion was adequate because data were oversampled.

Tracker3D then entered an 'editing' step in which the user could review the reconstructed paths and, if necessary, make corrections. Most often, because the $2 \mathrm{D}$ reconstruction step used very conservative path parameters, the user would simply join fragments of a trajectory into a single longer path. Whenever there was any doubt about whether to join fragments, we watched the original video on a 14" TV monitor to help make our decision.

Tracker3D then entered the 3D reconstruction step, in which paths from both cameras were combined to triangulate trajectories in 3 dimensions. The criteria for associating paths between cameras were upper limits to distances of closest approach between the rays through physical space from paths in the 2 views, together with limits on plausible fish velocity between successive frames.

Details of the Tracker3D algorithms are available at www.ocean.Washington.edu/people/faculty/grunbaum/ Tracker3D. The output from Tracker3D was a simple ASCII text file containing an identifier for each fish and its 3D $(x-y-z)$ position. To reduce high-frequency noise due to frame-rate oversampling, the output file was then passed through a filtering program written in Perl that reduced the frame rate to $5 \mathrm{~Hz}$. We used the filtered out- put to compute the polarity and speed of fish groups over time.

Computer simulation experiments. Basic model: We constructed a 3D model of fish schooling based loosely on preliminary measurements of danio movements taken from early film trials. Note that we did not attempt to perfectly emulate the real-world behavior of danios. Rather, we were interested in using computer simulations to inform us on general self-organization principles. Therefore, although our simulated fish are reasonably realistic, they are not perfectly so (e.g. gravity is not included). However, our simulations are a more physically realistic extension of existing sumof-forces fish school models (e.g. Aoki 1982, Huth \& Wissel 1990, Romey 1996, Couzin et al. 2002).

We set maximum speed at 12 body lengths (BL) s ${ }^{-1}$, and the maximum force $\left(F_{\max }\right)$ was set to impart a $12 \mathrm{BL} \mathrm{s}^{-2}$ acceleration on the fish, reflecting limits we observed in a pilot study. Since real danios are ca. $5.3 \mathrm{~cm}$ long and have a mass of $1.7 \mathrm{~g}$ (see 'Biological observations' above), $F_{\max }=102$ dynes. Although real danios are laterally compressed, our model fish were cylindrical for simplicity, with $1 \mathrm{BL}=5.3 \mathrm{~cm}$, and a body diameter $0.15 \mathrm{BL}$. Finally, because most fish have a rear blind area (Aoki 1982), we imposed a maximum (forward) viewing angle $\theta= \pm 150^{\circ}$. Individuals outside the maximum viewing angle were ignored (Couzin et al. 2002).

Each simulation began at time $t=0$ with a population of 4 or 8 fish scattered randomly within a spherical volume scaled to $12 \pi \mathrm{BL}^{3}$ per fish, centered at the origin. Thus, starting density was constant regardless of population size. All fish started with a random velocity, uniformly distributed between 0 and $6 \mathrm{BL} \mathrm{s}^{-1}$. Individuals did not begin a simulation inside each other's body volume; however, during simulations such overlaps sometimes occurred. We considered these intrusions to be analogous to 'collisions' (i.e. mistakes in schooling behavior) and recorded when and where they occurred. Once the simulation began, the individuals were not constrained; the domain was empty and infinite. We used simulated population sizes of 4 and 8 fish so model results could be directly compared with the behavioral observations and ran each simulation for 1800 time steps $(60 \mathrm{~s}$ at $30 \mathrm{~Hz}$, so that each time step = $1 / 30 \mathrm{~s})$. Each set of simulation conditions was replicated 15 times.

Behavioral movement rules: Many simulations use forces that accelerate the masses of individual fish to represent behavior (Aoki 1982, Niwa 1994, 1996, Romey 1996). The strength and direction of those forces are taken to implicitly reflect the consequences of complex sequences of events, including sensory perception, cognitive and reflexive processing, and swimming biomechanics. In this paper, we assumed all 
model fish were subject to only 2 force vectors: the 'social force' $\left(\mathbf{S}_{i}\right)$ and the 'random force' $\left(\mathbf{R}_{i}\right)$ on each fish $i$ during time $t$. The social force likely depends on many parameters in real animals: (1) the number of influential neighbors (Warburton \& Lazarus 1991), (2) the shape of the attraction/repulsion function (Romey 1996), (3) the strength of the 'alignment' force (Sannomiya \& Duostari 1996), (4) the width of attraction, repulsion, and alignment regions (Couzin et al. 2002), and (5) the total population size (Flierl et al. 1999). In this paper, we explore the strength of the alignment force, and how it affects certain emergent properties of fish schools (e.g. polarity, group speed). We consider other factors elsewhere (Viscido et al. unpubl.).

To determine the behavioral decision of a fish, we first defined a total force vector $\left(\mathbf{F}_{i}\right)$ on any individual $i$ during time $t$ as a sum of social and random forces (Inagaki et al. 1976, Matuda \& Sannomiya 1980, Aoki 1982, Niwa 1994, Sannomiya \& Duostari 1996):

$$
\mathbf{F}_{i}=\mathbf{S}_{i}+\mathbf{R}_{i}
$$

If the computed magnitude of $\mathbf{F}_{i}$ exceeded $F_{\text {max }}$, the simulation program re-scaled $\mathbf{F}_{i}$ to have magnitude $F_{\max }$ (while preserving direction). Note that, in addition to random and social force, real fish will also experience an opposing force due to drag. For simplicity, we did not include drag in this model; the effects of drag are reported elsewhere (Viscido et al. unpubl.).

Social force: The total social force acting on fish $i$ during time step $t$ was the sum of social forces between fish $i$ and each other member of the population:

$$
\mathbf{S}_{i}=\sum_{j=1}^{\rho-1} \mathbf{S}_{i j}
$$

where $\rho$ is population size ( $\rho=4$ or 8 ), and $\mathbf{S}_{i j}$ represents the social force between fish $i$ and each neighbor $j$. The social force, consisted of 2 distinct components: the attraction/repulsion force and the alignment force. Fish had a preferred distance to their neighbors $\left(\delta_{p}=\right.$ $1.9 \mathrm{BL})$ based on observed preferred distances for Chromis punctipinnis (Parrish \& Turchin 1997). We defined a neighbor fish $j$ that was within $\pm 0.5 \mathrm{BL}$ of the preferred distance as being within the 'alignment zone' (Fig. 1a). Within the alignment zone, fish $i$ experienced an alignment force due to $j\left(\mathbf{A}_{i j}\right)$ in the swimming direction of fish $j$. We varied the magnitude of vector $\mathbf{A}_{i j}$ from 0.5 to $50 \%$ of $F_{\max }$ (i.e. always far less than the maximum possible attraction/repulsion force) in separate model runs. The magnitude of $\mathbf{A}_{i j}$ was constant throughout the alignment zone, and for all individuals in a given simulation experiment (Fig. 1b).

Outside the alignment zone, fish $i$ was either attracted to, or repulsed from, fish $j$, depending on the distance between them. When fish $j$ was closer than (a)

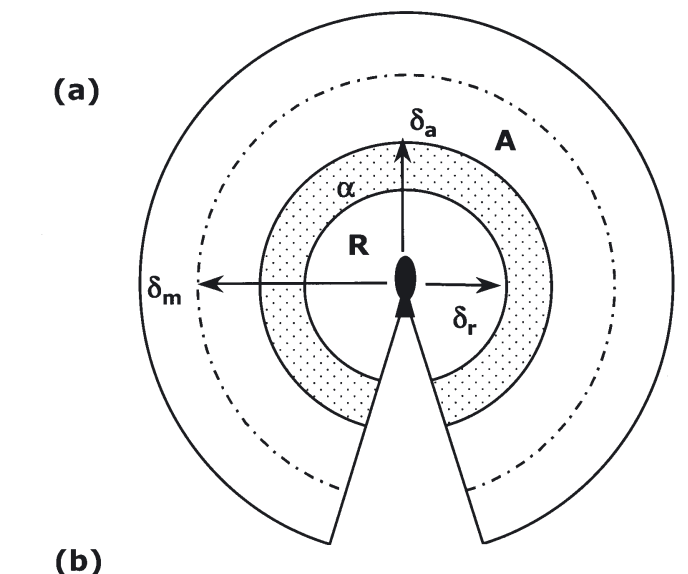

(b)

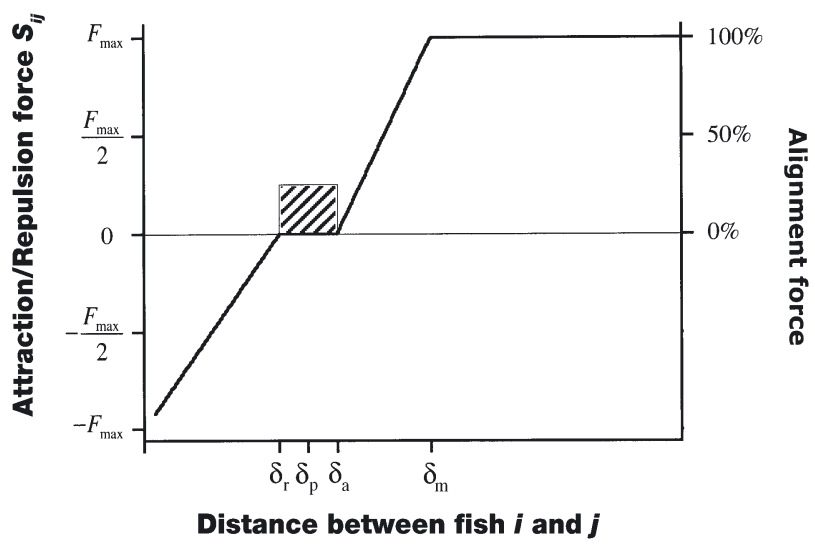

Fig. 1. Social attraction and alignment forces used in the simulation model. (a) The 3 regions over which social forces act: the repulsion zone $(R)$, the alignment zone $(\alpha)$, and the attraction zone (A). Arrows point to the distance at which repulsion begins $\left(\delta_{\mathrm{r}}\right)$, the distance at which attraction begins $\left(\delta_{\mathrm{a}}\right)$, and the distance of maximum attraction $\left(\delta_{\mathrm{m}}\right)$. The $60^{\circ}$ arc behind the fish is the 'blind spot.' (b) The 2 forces over distance, with social (attraction/repulsion) force (thick line) in units of dynes, and alignment force (shaded area) as a percent of maximum force $\left(F_{\max }\right)$. For simplicity only the $25 \%$ alignment force is shown. $\delta_{\mathrm{p}}$ : preferred distance to neighbor

the repulsion distance $\delta_{\mathrm{r}}$, it was in the 'repulsion zone' (Fig. 1a), and the force acting on fish $i$ due to fish $j$ was directed along the vector from $j$ to $i$. This 'repulsion force' increased linearly from $-F_{\max }$ at a distance of 0 from fish $i$, to 0 at exactly $\delta_{\mathrm{r}}=1.4 \mathrm{BL}$ (Fig. $1 \mathrm{~b}$ ). When fish $j$ was farther away than the attraction distance $\delta_{\mathrm{a}}$ fish $j$ was in the 'attraction zone' (Fig. 1a), and the force acting on fish $i$ due to fish $j$ was directed along the vector from $i$ to $j$. This 'attraction force' increased linearly from 0 at exactly $\delta_{\mathrm{a}}=2.4 \mathrm{BL}$ to a maximum of $+F_{\max }$ at a distance of 5 BL (Fig. 1b). The social force remained at $+F_{\max }$ to a distance of $100 \mathrm{BL}$ (except within the blind region), at which point fish $j$ was assumed to be outside the visual range of fish $i$ (and hence the social force was 0). 
Random force: During each time step, a variable random force acted on each simulated fish, representing stochastic behavioral and environmental factors that were not explicitly modeled (Flierl et al. 1999). This random force was a vector with random direction and whose magnitude was a random normal variate, with a mean of 0 and a standard deviation of $F_{\max } / 6$ (i.e. 17 dynes). Because there was an upper limit to total force on an individual $\left(F_{\max }\right)$, this random force was a relatively smaller component of total force when social forces were large, such as when a fish was about to collide with a neighbor, or was separated from the school. Conversely, the random force was a relatively larger component of total force when social forces were small, such as when all neighbors were in the alignment zone (where forces were always $50 \%$ of attraction/repulsion or less), or when no neighbors were present within the maximum sensing distance (where social force was 0).

Statistical analysis. Because our observational experiments lasted a relatively long time (10 $\mathrm{min})$, and therefore fish were likely to encounter the aquarium edge frequently during an observation, we tested whether our metrics might have been affected by the group's proximity to an edge surface. We compared the distance between the group center and the nearest tank edge, in each frame, to each of the 3 schooling metrics tested here, using a product-moment correlation. There was no relationship between edge proximity and any of the metrics used ( 4 fish: $\mathrm{r}^{2}$ polarity $=0.03$, $\mathrm{r}^{2}$ speed $=0.02, \mathrm{r}^{2}{ }_{\mathrm{NND}}=0.05 ; 8$ fish: $\mathrm{r}^{2}$ polarity $=0.01, \mathrm{r}_{\text {speed }}{ }^{2}$ $0.01, \mathrm{r}^{2}{ }_{\mathrm{NND}}=0.03 ; \mathrm{p}>0.05$ in all cases).

We report statistical results for 3 emergent properties of both real and simulated fish schools: polarity, group speed, and mean NND. Polarity and group speed are characteristics of the group (not the individual), and we therefore computed a single value of each property for each group at each time step. Fish were defined as being in a group if they were within $5 \mathrm{BL}$ of at least 1 other neighbor, and otherwise were considered stragglers (and, hence, not included in the group property analysis). When more than 1 group was present during a single time step (e.g. 2 groups of 4 fish in an 8 fish trial), we computed the average value across all groups, to obtain a single mean polarity and mean group speed for each time step. These group-based means were not 'weighted' in any way (e.g. by group size). NND is a property of individuals, and we therefore computed a single mean NND value for the entire population at each time step. Additional metrics at the individual, group, and population level, including path curvature, group size, and collision rate, are reported elsewhere (Viscido et al. unpubl.). We also display the polarity and group speed of a single group in several places (see Figs. $2 \& 3$ ). This second approach is merely a visual mechanism to demonstrate the observed group-level patterns (which would not be visible if 'averaged out'), and was not used for computing timeaveraged statistics. In these cases only (see Figs. 2 \& 3), we report the maximum value obtained by any group within the population for each time step.

Time averages of the 3 properties were computed for each single $30 \mathrm{~s}$ segment of a real fish trial. For simulations, we used the latter half (time steps 900 to 1800). This choice was designed to (1) make the simulation time period and the filmed time period comparable, and (2) eliminate the effects of initial conditions (i.e. reduce simulation artifacts). We also compared polarity and group speed using a correlation analysis.

Polarity: We estimated group polarity as the mean vector angle deviation between group and individual heading (Huth \& Wissel 1992). Let $\mathbf{V}_{i}$ represent a unit velocity vector for fish $i$ (scaled to preserve direction) and let $\mathbf{U}$ represent the unit velocity vector for the group's center. We compute the 3D angle deviation $\alpha_{i}$ between each fish's velocity vector and that of the group, by taking the inverse cosine of the dot product:

$$
\alpha_{i}=\cos ^{-1}\left(\mathrm{~V}_{i} \cdot \mathrm{U}\right)
$$

The group's polarity $\phi$ is then the mean of these angle deviations:

$$
\phi=\frac{1}{G} \sum_{i=1}^{G} \alpha_{i}
$$

where $G$ represents the group size. Note that as $\phi$ approaches $0^{\circ}$, fish headings approach parallel, whereas when polarity approaches $90^{\circ}$, fish headings approach perpendicular. Because this metric is counter-intuitive (high numbers represent a low amount of polarity), we used a non-dimensionalized form of polarity $\left(\phi^{*}\right)$ defined as:

$$
\phi^{*}=\frac{\left(90^{\circ}-\phi\right)}{90^{\circ}}
$$

The non-dimensionalized polarity $\phi^{*}$ thus took on values ranging from 0 (completely non-polarized) to 1 (perfectly aligned). Note that this approach computes the polarity for a single group of fish; this measure was reported either as the maximum observed for all groups within the population (see Fig. 2), or as the mean across all groups within the population (see Figs. 4, 6 \& 7).

Group speed: Group speed $v_{t}$ in a single time step was computed as the magnitude of the group centroid's velocity vector from time $t$ to $t+1$. The speed was calculated in units of $\mathrm{cm}$ frame $\mathrm{e}^{-1}$ for real fish and BL time step $^{-1}$ for simulated fish and converted to $\mathrm{cm} \mathrm{s}^{-1}$ and $\mathrm{BL} \mathrm{s}^{-1}$, respectively. For ease of comparison between real and simulated data, we used a nondimensionalized form of group speed $\left(V^{*}\right)$ computed as: 


$$
V^{*}=\frac{V_{t}}{V_{\max }}
$$

where $V_{\max }$ represents the maximum speed achieved across all replicates. For real fish, $V_{\max }$ represented the maximum for an entire trial (5 min); for simulated fish, $V_{\text {max }}$ represented the maximum for all runs for each set of simulation conditions. The non-dimensionalized group speed $V^{*}$ therefore ranged from 0 (stationary) to 1 (moving at the maximum velocity observed for that set of experimental conditions). Note that this approach computes the speed for a single group of fish; this measure was reported either as the maximum observed for all groups within the population at each time step (see Fig. 3), or as the mean across all groups within the population (see Figs. $6 \& 7$ ).

Nearest-neighbor distance: The NND for any fish $i$ $\left(\mathrm{NND}_{i}\right)$ was the minimum distance $d_{i, j}$ between fish $i$ to all neighbors $j$ in the population $\rho$ (Krebs 1989):

$$
N N D_{i}=\min \left(d_{i, 1}, d_{i, 2}, \ldots, d_{\rho}\right)
$$

We report time-averaged NND values for each simulation run ( $\mathrm{N}=15$ for each population size and alignment force strength) and each real fish trial ( $\mathrm{N}=3$ for each population size). For real fish, we used $1 \mathrm{BL}=5.3 \mathrm{~cm}$ to obtain NND in units of BL. Note that computing NND in this fashion means that, in some cases, the same distance will be counted twice when there is a reflexive pair (e.g. $A$ is the closest neighbor to B, and vice versa).

\section{RESULTS}

In our observations, the maximum observed polarity $\left(\phi^{*}\right)$ of all giant danio schools within the population varied over the full possible range from 0 (nonpolarized) to 1 (perfectly aligned) throughout each trial for both 4- and 8-fish populations (Fig. 2a,b). Fish schools in populations of 4 individuals had a mean polarity across all trials $(\mathrm{N}=3)$ of $0.57( \pm 0.08 \mathrm{SD})$, whereas schools within 8-fish populations $(\mathrm{N}=3)$ had a mean polarity of $0.41( \pm 0.11 \mathrm{SD})$. Group speeds $\left(v_{t}\right)$ for schools within 4- and 8-fish populations were dynamic, ranging from 0 (stasis) to $11.6 \mathrm{~cm} \mathrm{~s}^{-1}$ (maximum) within $60 \mathrm{~s}$ (Fig. 3a, b). Mean speed was $7.35 \mathrm{~cm} \mathrm{~s}^{-1}$ ( \pm 1.79 S.D.) and $5.17 \mathrm{~cm} \mathrm{~s}^{-1}( \pm 2.39 \mathrm{SD})$ for 4 - and 8 -fish populations, respectively. However, average relative (non-dimensionalized) speed was very similar for both 4 fish $\left(V^{*}=0.64 \pm 0.15 \mathrm{SD}\right)$ and 8 fish $\left(V^{*}=0.56 \pm\right.$ $0.26 \mathrm{SD})$ groups. NND was not affected by population size (2-sample $t$-test, $\left.t_{s}=0.715, \mathrm{p}>0.25\right)$. Mean NND was $12.3 \mathrm{~cm}( \pm 6.6 \mathrm{SD})$ (i.e. $2.3 \pm 1.2 \mathrm{BL}$ ) for 4 fish and $16.8 \mathrm{~cm}( \pm 19.6 \mathrm{SD})$ (i.e. $3.1 \pm 1.8 \mathrm{BL})$ for 8 fish.

By comparison, ranges of polarity in simulated fish schools depended strongly on both the population size

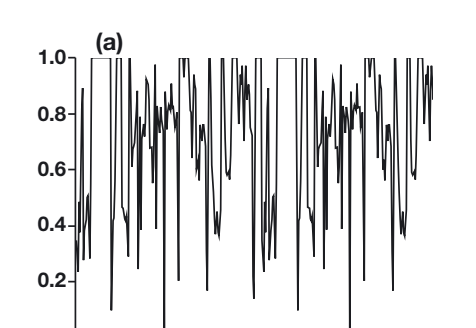

(b)

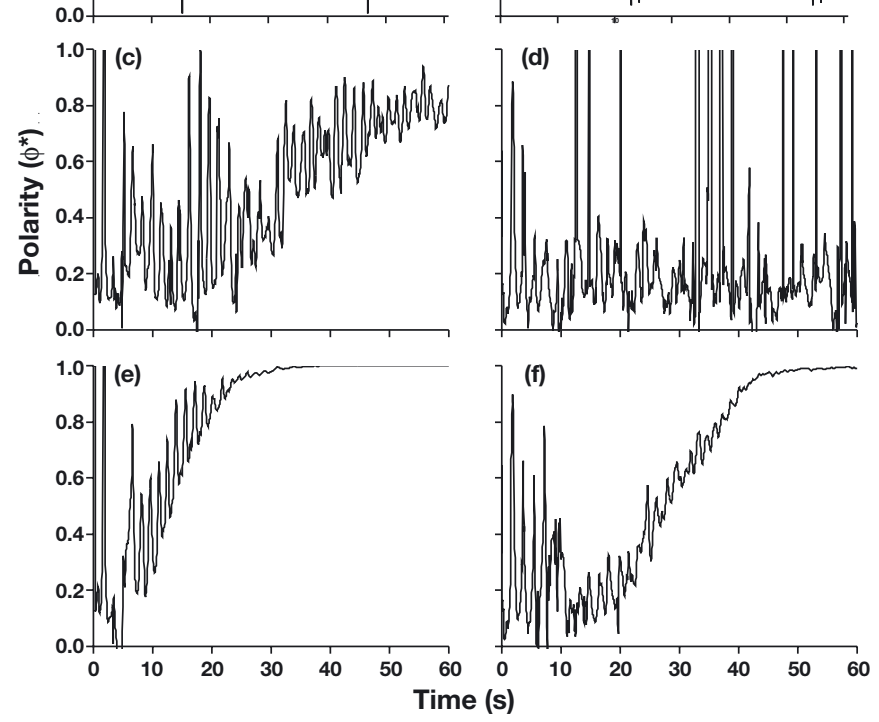

Fig. 2. Maximum observed polarity $\left(\phi^{*}\right)$ across fish schools within a single trial over time for populations of (a) 4 real fish, (b) 8 real fish, (c) 4 simulated fish with an alignment force of $1 \%$ of $F_{\max }$ (d) 8 simulated fish with an alignment force of $1 \%$ of $F_{\max }$ (e) 4 simulated fish with an alignment force of $10 \%$ of $F_{\max }$ and (f) 8 simulated fish with an alignment force of $10 \%$ of $F_{\text {max }}$

and the amount of alignment force (Fig. 2c-f). Even very small amounts of alignment force (e.g. $1 \%$ of $F_{\text {max }}$ ) caused schools within populations of 4 model fish to trend towards perfect alignment (Fig. 2c). Increasing population size, even to just 8 model fish, destroyed this alignment (Fig. 2d). For the stronger alignment forces used (e.g. $10 \%$ of $F_{\text {max }}$, Fig. 2e, f), schools always tended to near-perfect alignment, regardless of population size.

We quantified the relative effects of population size and alignment force strength on $\phi^{*}$ using a 2-way ANOVA across all simulation runs. The 1-way comparisons were highly significant $(\mathrm{df}=1, F=247.20, \mathrm{p}<$ 0.001 for population size; $\mathrm{df}=6, F=328.24$, $\mathrm{p}<0.001$ for alignment force), indicating that both population size and alignment force affect alignment. Furthermore, the 2-way population size $\times$ alignment force interaction was also highly significant (df $=6, F=$ 32.65, $\mathrm{p}<0.001$ ), indicating that the population size affects how sensitive polarity is to alignment force.

To estimate the relative amount of alignment force real fish might experience to produce these observed 


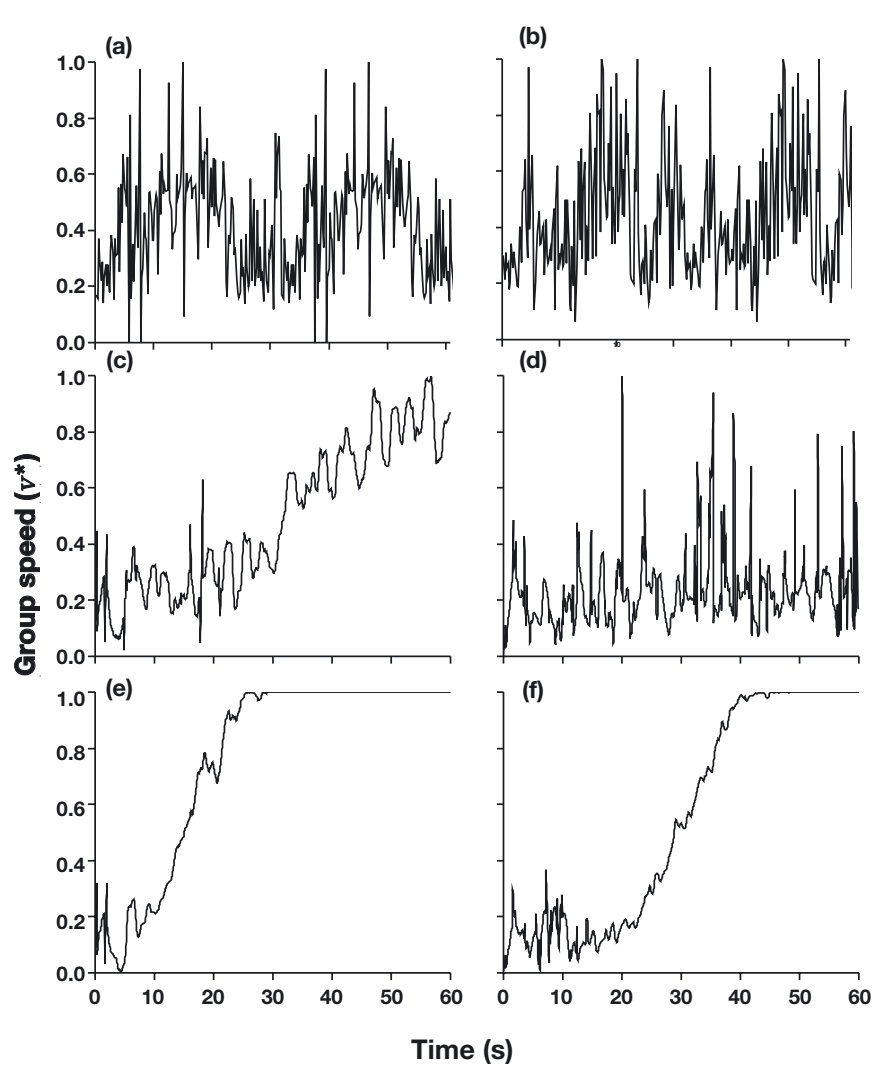

Fig. 3. Maximum observed group speed $\left(v^{*}\right)$ across fish schools within a single trial over time for populations of (a) 4 real fish, (b) 8 real fish, (c) 4 simulated fish with an alignment force of $1 \%$ of $F_{\max }$ (d) 8 simulated fish with an alignment force of $1 \%$ of $F_{\max }$ (e) 4 simulated fish with an alignment force of $10 \%$ of $F_{\text {max }}$ and (f) 8 simulated fish with an alignment force of $10 \%$ of $F_{\max }$

polarities, we plotted mean polarity for model fish across all replicates for each alignment force used, essentially creating a 'standard curve' (Fig. 4a). The mean polarity observed for schools in populations of 4 live fish was similar to simulations where the alignment force was 1 to $2 \%$ as strong as $F_{\max }$ (Fig. $4 \mathrm{a}$ ), whereas the mean polarity observed for schools in populations of 8 live fish was most similar to simulations where the alignment force was just above $5 \%$ of $F_{\max }$ (Fig. 4b).

Schools in model populations of 4 fish quickly reached maximum speed $\left(12 \mathrm{BL} \mathrm{s}^{-1}\right)$, with the time course of equilibrium exacerbated by increasing the alignment force strength (Fig. 3c,e). Increasing population size to 8 individuals damped this sensitivity, resulting in a relative group speed that was a fraction of the maximum observed $\left(v^{*}=0.17 \pm 0.07 \mathrm{SD}\right.$ at $1 \%$ alignment, Fig. 3d), a pattern visually similar to real fish (e.g. Fig. 3b). However, increasing the alignment force to $10 \%$ destroyed this similarity (Fig. 3f).

To quantify the relative effects of population size and alignment force strength on $v^{*}$, we performed a 2-way

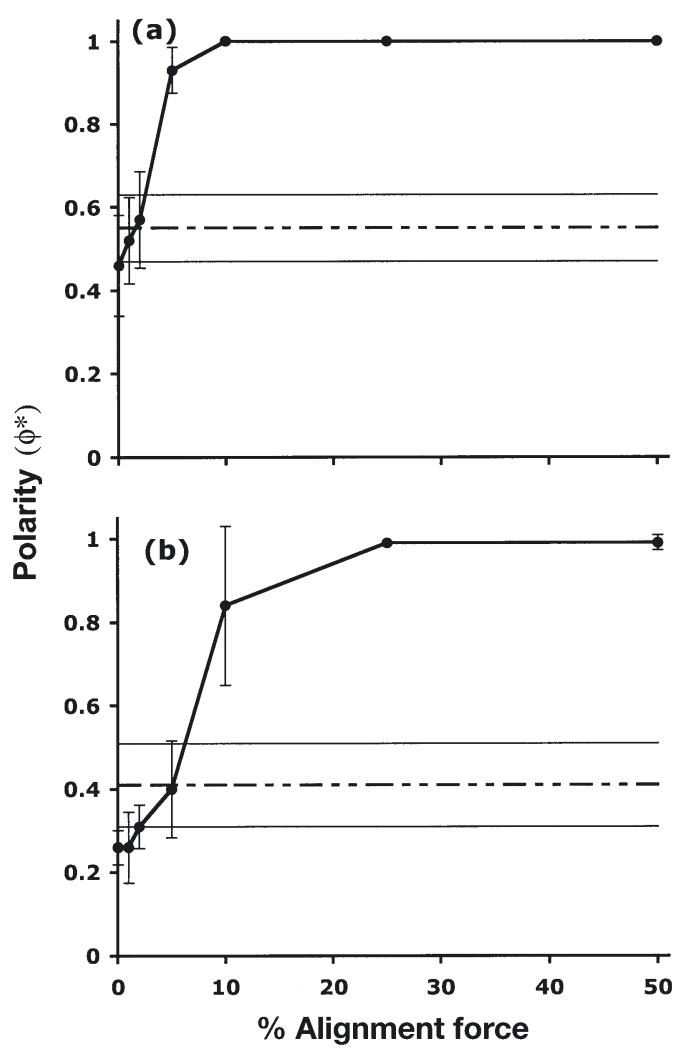

Fig. 4. Mean time-averaged polarity $\left(\phi^{*}\right)$ as a function of the alignment force strength, across all simulation experiments, for populations of (a) 4 and (b) 8 individuals. Alignment force strength is represented as a percentage of the maximum attraction/repulsion force $F_{\max }$. For reference, the overall mean polarity for real fish is shown (dashed line) $\pm 1 \mathrm{SD}$ (thin lines)

ANOVA across all simulation runs. As with polarity, 1 -way comparisons were also highly significant $(\mathrm{df}=1$, $F=193.34, \mathrm{p}<0.001$ for population size; $\mathrm{df}=6, F=477.98$, $\mathrm{p}<0.001$ for alignment force), indicating that both population size and alignment force have strong effects on group speed. The 2-way population size $\times$ alignment force interaction for $v^{*}$ was also highly significant $(\mathrm{df}=6$, $F=40.66, \mathrm{p}<0.001$ ), indicating that population size affects how sensitive group velocity is to alignment force.

To deduce the relative amount of alignment force real fish might experience to produce observed NND values, we plotted mean NND for model fish across all replicates for each alignment force used, similar to the 'standard curve' created for polarity (Fig. 5). The mean NND observed in 4-fish populations, was similar to simulations where the alignment force was 1 to $2 \%$ as strong as $F_{\max }$ (Fig. 5a), whereas the mean NND observed in populations of 8 live fish was considerably higher in all cases than that observed in simulated schools (Fig. 5b). In general, however, the mean NND for simulated fish fell within \pm 1 SD of that observed for real fish, regardless of the alignment force used. 


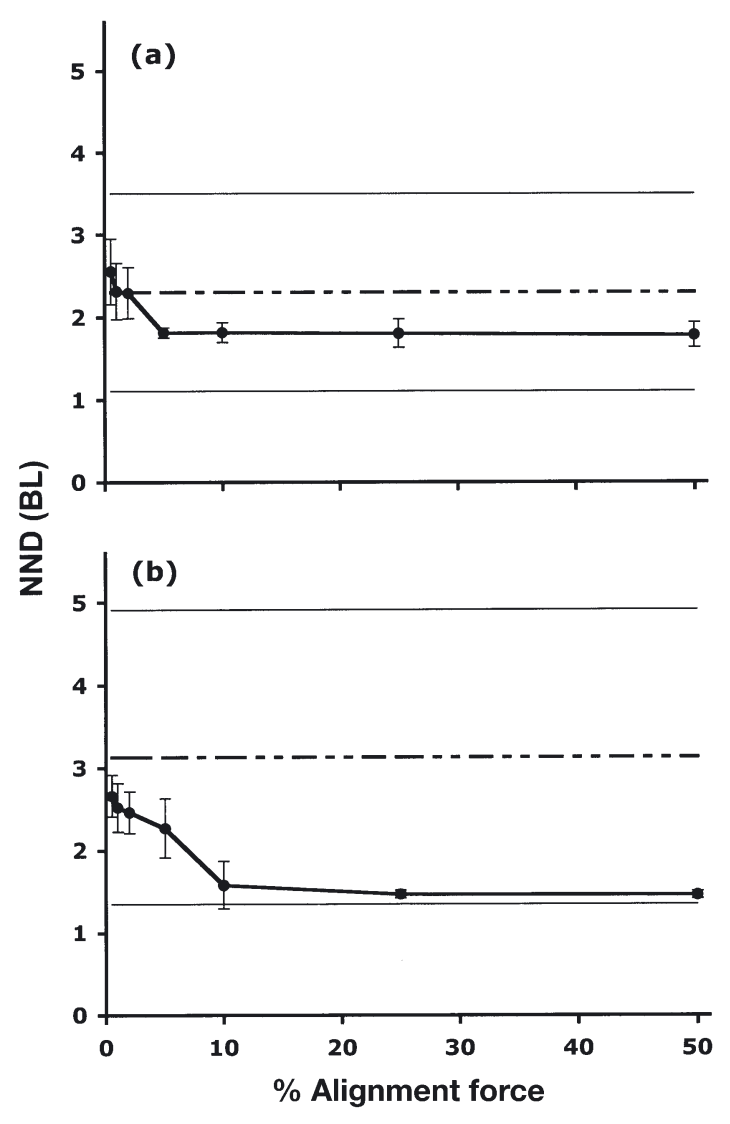

Fig. 5. Mean time-averaged nearest-neighbor distance (NND) as a function of the alignment force strength, across all simulation experiments, for populations of (a) 4 and (b) 8 individuals. Alignment force strength is represented as a percentage of the maximum attraction/repulsion force $F_{\max }$. For reference, the overall mean NND for real fish is shown (dashed line) $\pm 1 \mathrm{SD}$ (thin lines)

To quantify the relative effects of population size and alignment force strength on NND, we performed a 2-way ANOVA across all simulation runs. As with the other metrics, the 2-way population size $\times$ alignment force interaction for NND was highly significant ( $\mathrm{df}=$ $6, F=13.42, \mathrm{p}<0.001$ ), indicating that the value of each factor can change the NND that would otherwise be observed due to the other factor. However, when considering the 1-way effects, NND was significantly affected by alignment force alone ( $\mathrm{df}=6, F=477.98$, $\mathrm{p}<0.001)$, but not by population size alone $(\mathrm{df}=1, F=$ $0.0, \mathrm{p}>0.95)$. Thus, results with simulated fish reflected the results with real fish: population size, by itself, was not important in determining NND.

Correlation analysis showed a positive relationship between $v^{*}$ and $\phi^{*}$. For populations of 4 live fish, there was a moderate positive correlation between the 2 properties $\left(\mathrm{r}^{2}=0.42, \mathrm{p}<0.01\right.$, Fig. 6$)$, while in populations of 8 live fish there was a stronger positive correlation $\left(r^{2}=0.67, p<0.001\right.$, Fig. 6). The relationship was even more dramatic $\left(\mathrm{r}^{2}=0.98\right.$ and $0.99, \mathrm{p}<0.001$ in both cases) for simulated fish regardless of population size (Fig. 6). For real fish, the position of the time-averaged values along the $V^{*}-\phi^{*}$ axes depended partly on the individual fish in the school: each 8-fish trial had a 'characteristic' set of values (Fig. 6c), for example, suggesting that emergent properties depend very strongly on the quirks of the individual school members. For simulated fish (populations of which were always entirely identical), the position of the time-averaged values along the $V^{*}-\phi^{*}$ axes depended entirely upon the strength of the alignment force (Fig. 6b,d).

\section{DISCUSSION}

Our results quantify the relationship between the speed of motion and polarity of fish schools, and directly compare emergent properties displayed by real fish with those displayed in simulation. The similarity of simulated fish school behavior to that of real fish depended strongly on the alignment force strength of the former (Figs. 4 \& 5).

Real fish schools showed a wide range of polarity values over time (Fig. 2), from completely non-polarized

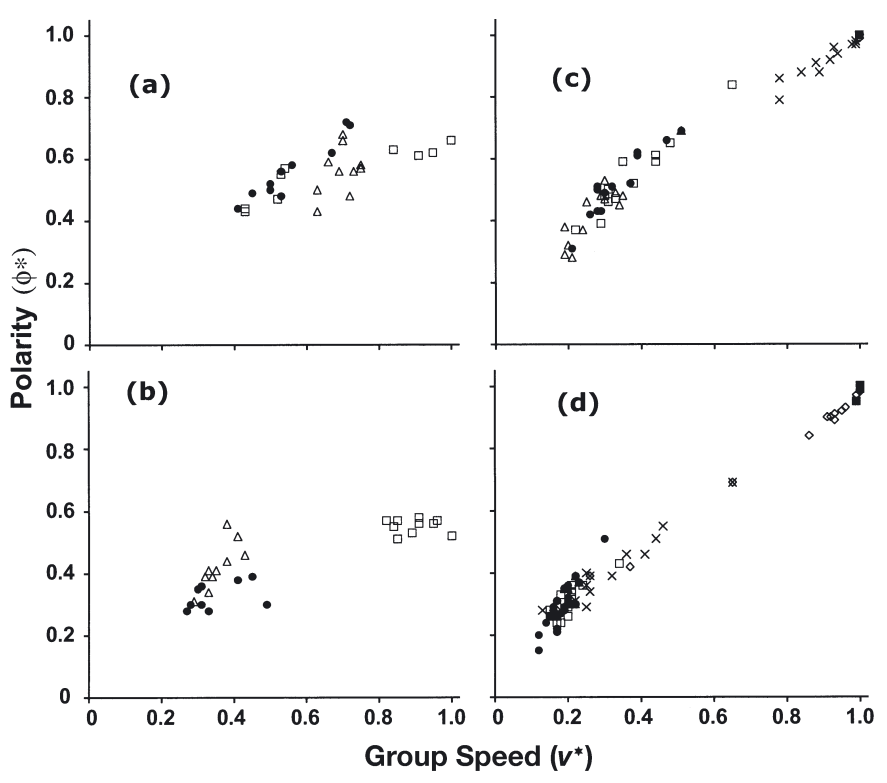

Fig. 6. Relationship between time-averaged group speed $\left(V^{*}\right)$ and time-averaged polarity $\left(\phi^{*}\right)$ for simulation experiments with (a) 4 real fish, (b) 8 real fish, (c) 4 simulated fish, and (d) 8 simulated fish. Polarity $\phi^{*}$ is expressed as a function of group speed. For real fish $(a, b)$, each point represents a $30 \mathrm{~s}$ time average, with triangles, squares, and circles representing the 3 different trials for that population size. For simulated fish $(\mathrm{c}, \mathrm{d})$, each point represents the $30 \mathrm{~s}$ time average for a single simulation run, with symbols representing percent of alignment force: $50 \%(\boldsymbol{\bullet}), 25 \%(\mathbf{\Delta}), 10 \%(\diamond), 5 \%(\times), 2 \%$ $(\bullet), 1 \%(\square)$, and $0.5 \%(\Delta)$ 
(i.e. a 'swarm,' Shaw 1978) to perfectly aligned (i.e. a 'school,' Shaw 1978). Inspection of school polarity time series reveals that real fish schools were very strongly aligned most of the time, with irregular periods during which this orderly structure was destroyed and the school became a disorganized swarm (e.g. Fig. 2b). For real fish, therefore, an aligned school appears to be rather like a self-organized critical state, which is dynamically stable until something happens that destroys the self-organization (Bak et al. 1988, Wu \& David 2002) — such as a single individual abruptly changing position, forcing other group members to re-shuffle. Schools within populations of 8 simulated fish with an alignment force of only $1 \%$ of $F_{\max }$ showed the opposite pattern, spending most of their time in a disorganized swarm, and then occasionally becoming a polarized school (e.g. Fig. 2d). However, if the alignment forced was substantially increased (to $10 \%$ or higher), continuous polarization resulted (Fig. 2f).

Theoretical studies have indicated that there may be a few dynamically stable group types, and that intermediate group types may be relatively unstable. For example, Couzin et al. (2002) found several dynamically stable collective behaviors - the swarm, the torus, and the parallel group - and predicted that animal groups should change rapidly between these 3 states, but spend little time in the intermediate states. Our findings agree with this prediction, for both real fish (where the stable state is a parallel group, Fig. 2a) and model fish (where the stable state is either a swarm or a school, Fig. 2e). In such dynamically stable configurations, transitions to another configuration are probably the result of changes in individual behavior (Couzin et al. 2002).

In model fish, polarity depended strongly on the population size as well as on the strength of the alignment force (Fig. 4). Even weak alignment strength (e.g. 1\%) drove schools in 4-fish populations to perfect polarity, but it caused schools in 8-fish populations to enter a dynamically stable swarm configuration (Fig. 2). Couzin et al. (2002) showed convincingly that attraction and orientation zone widths are critical factors in determining group structure, and we hypothesize that the relative size of the zones we used played a key role in the differences observed between 4 - and 8-fish populations. In a 4 -fish population, all individuals could conceivably be in each other's alignment zone (Fig. 1), allowing rapid and unswerving alignment. In an 8-fish population, on the other hand, at least 1 neighbor will almost always lie outside each individual's alignment zone, leading to increasing disorganization as fish turn toward or away from those in the other zones. If our hypothesis is correct, then 4-fish model populations can reach a dynamically stable state, and 8-fish model populations can have their stable state changed from swarm to parallel school, by changing the size of 1 or more zones.
To date, most other zone-based models implicitly assume that the alignment force is equal in magnitude to the attraction/repulsion force (Aoki 1982, Huth \& Wissel 1990, Hiramatsu et al. 2000, Inada \& Kawachi 2002). As we have shown in this paper, alignment forces that are high relative to attraction/repulsion forces are likely to produce schools that have artificially high and invariable alignment (Figs. $2 \& 4$ ). Additionally, the little available experimental evidence indicates that such a high alignment force is unlikely in real fish. Using tank experiments to estimate the parameters of a zone-based model, Duostari \& Sannomiya (1995) and Sannomiya \& Duostari (1996) calculated the alignment force as being roughly $1 / 3$ to $1 / 2$ the strength of the attraction/repulsion force. Our own tank observations indicated that the timeaveraged polarity of real fish schools is similar to that of simulated schools whose members experience an alignment force around 1 to $2 \%$ of the attraction/repulsion force's magnitude. However, with few exceptions (e.g. Hiramatsu et al. 2000) the polarity of real fish schools is rarely reported. Our results also indicate that timeaveraged group statistics may be misleading, particularly when the group is rapidly transitioning between 2 very different states (Fig. 2).

Interestingly, while both group-level metrics examined here (polarity and group speed) varied depending on population size for simulated fish, the individuallevel metric (NND) did not, either for real fish, or for simulated fish (Fig. 5). Indeed, simulated fish approached very nearly the preferred NND defined in the model, and they were increasingly good at maintaining that distance as alignment force increased. Simulated schools with higher alignment forces more perfectly approached the preferred NND because they were more perfectly polarized (Fig. 4), and members of polarized schools will be more likely to maintain a constant distance from one another. For real fish, NND may depend on fish body shape or sensory apparatus. For example, fish that have been blinded or had their lateral line damaged can still school, but their average NND is different from fish with all sensory systems intact (Pitcher et al. 1976).

For both real and simulated fish, group speed $\left(V^{*}\right)$ and group polarity $\left(\phi^{*}\right)$ were positively correlated (Fig. 6). This implicitly makes sense: when the group is not aligned, individual members are facing in many different directions, and their velocity vectors will tend to cancel each other out, leading to little net movement for the group as a whole. It is also difficult, if not impossible, for non-aligned individuals to move very far without colliding with one another. On the other hand, when the group is highly aligned, individual members are facing in approximately the same direction, and this can lead to rapid group movement in that direction. To our knowledge, the relationship between 
group speed and velocity has not been investigated in previous models, although the experimental results of Hiramatsu et al. (2000) suggest a relationship.

Our results suggest certain ranges of state space over which animal populations can sustain a regular architecture. We imagine a 3D state space where each emergent property statistic measured in this study (NND, group speed, and polarity) is represented along an axis, and within which different group types can be placed (Fig. 7). The simple behavioral rules we used in this study (zone-based attraction, repulsion, and alignment, with only other group-mates as stimuli) lead to a certain subset of possible architectures. The relationship between polarity, group speed, and NND was extremely strong in our simulations (2-way regression, $\left.\mathrm{r}^{2}=0.98, \mathrm{p}<0.001\right)$, and followed the functional form:

$$
\phi^{*}=0.25 V^{*}-0.61(\mathrm{NND})+1.84
$$

(indicated by the meshed plane, Fig. 7). Thus, if group types were observed in a different region of the state space, we would imagine other behavioral 'forces' were at work. For instance, in the Gulf of California, immense herring schools (hundreds of thousands) tend to hover in space, with group speed effectively zero, but are aligned almost perfectly (Parrish 1992). The herring school in this case is subject to a major force (predation risk) not considered in our simple attraction/repulsion/alignment scheme, the defense against which is to remain in polarized schools to facilitate escape (Parrish 1992). We hope to improve the realism of our model in the future by including factors such as internal state and predation

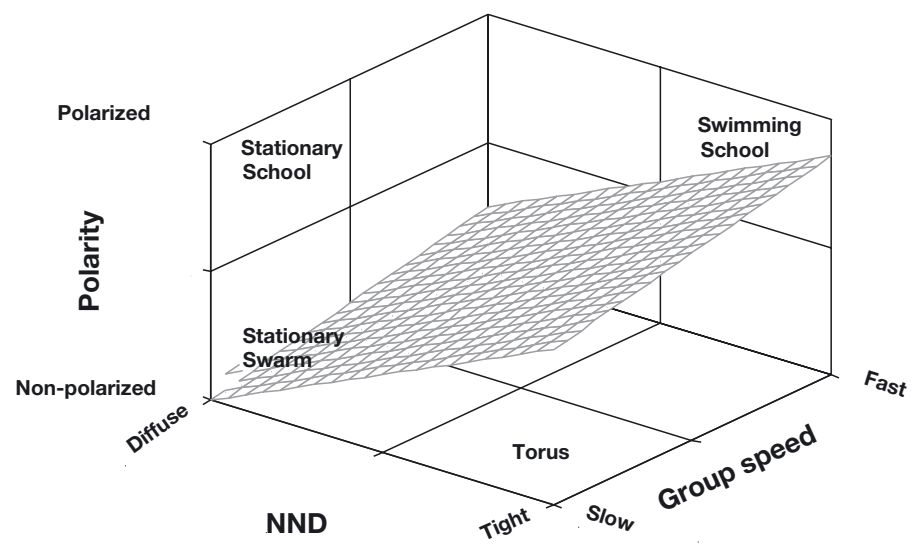

Fig. 7. Relationship between 3 different group properties: polarity, nearest-neighbor distance (NND), and group speed. The plane depicts a 2 -way regression of polarity against NND and group speed for our model data shown in Eq. (8). Hypothetical positions within the 3D state space are shown for several common types of aggregations: stationary swarm (e.g. krill swarms, Ritz 1994), stationary school (e.g. polarized schools of 'hovering' herring, Parrish 1992), torus (e.g. Fig. 1 in Parrish et al. 2002, Couzin et al. 2002), fountain effect (e.g. Pitcher \& Parrish 1993), and swimming school (e.g. swimming danio groups, Figs. 2 \& 3 ) risk, so that a greater diversity of group architecture types can be exhibited using a single set of simple rules.

The connection between different emergent properties such as group speed and polarity also suggests constraints on individual and group behavior: if the individual group members want the group to accelerate, for example, a viable strategy may be to increase their polarity or decrease their NND, such that the group moves to a different region of the 3D state space (Fig. 7). We also suspect that a few variable individuals can have a strong effect on group characteristics, and hence the position of the school in the 3D state space. For example, adding even a single individual whose movement rules differ from the other group members can change overall school structure and motion (Romey 1996). In the past, little attention has been given to how different emergent properties such as group speed and NND relate to one another. Instead, these properties are reported separately, and often only 1 or 2 properties are measured in a given study (reviewed in Parrish et al. 2002). Our research indicates that understanding the relationships between multiple emergent properties is an especially fruitful avenue for investigating group behavior. To further examine these questions, a more detailed version of the model is being implemented (Viscido et al. unpubl.). We are also conducting experiments with larger fish schools $(16,32)$, and in a larger experimental tank $\left(4 \mathrm{~m}^{3}\right)$, as well as adding perturbations to the system (e.g. artificial predators) to make it less static.

The coupling of observational and modeling efforts can produce insights neither technique would provide by itself (Bumann et al. 1997). For example, our observations with real fish showed how polarity and group speed changed over time, providing a basis for comparison with the simulation results. That comparison indicated that a relatively simple simulation model, including only a large social force, a small alignment force, and a small random force, can produce results approaching those of real fish (Figs. 2 \& 3). The model, in turn, suggested that the emergent properties observed in the live fish observations can only be achieved with a small alignment force. With too large an alignment force (above $5 \%$ of the total force), fish schools would be perfectly and invariably aligned, which they are not in real life (Fig. 2a,b), whereas with too small an alignment force, groups would be completely non-polarized (Fig. 4). Thus, our model indicates that, in the absence of other environmental factors (e.g. foraging behavior, predation avoidance), these schooling fish may primarily be concerned with maintaining the proper distance to their neighbors, and only secondarily concerned with alignment. How those other environmental factors would affect both model and real fish behavior is an important avenue of future investigation. 
Acknowledgments. This paper benefited from the assistance of many helpful individuals. J. Weitzman, B. Cheney, T. Mongillo, and I. MacFarland assisted with observations, fish care, and motion analysis. S. Menden-Druer helped produce the Tracker3D algorithms. T. Daniel, M. Kot, C. Bergstrom, T. Reluga, and the rest of the University of Washington's Mathematical Biology group provided several useful discussions on the analysis of both modeling and observational results. We also thank the participants of the Conference on Swarming Behavior at UCLA for useful feedback. Two anonymous reviewers gave insightful criticism that greatly improved the paper. Funding was provided by NSF grant CCR-9980058 to J.K.P. and D.G.

\section{LITERATURE CITED}

Aoki I (1982) A simulation study on the schoolng mechanism in fish. Bull Jpn Soc Sci Fish 48:1081-1088

Aoki I (1984) Internal dynamics of fish schools in relation to inter-fish distance. Bull Jpn Soc Sci Fish 50:751-758

Bak P, Tang C, Wiesenfeld K (1988) Self-organized criticality. Physiol Rev A 38:364-374

Bumann D, Krause J, Rubenstein D (1997) Mortality risk of spatial positions in animal groups: the danger of being in the front. Behaviour 134:1063-1076

Burger J, Gochfeld M (1991) The common tern: its breeding biology and social behavior, 1st edn. Columbia University Press, New York

Clark M, Irwig K, Wobke W (1997) Emergent properties of teams of agents in the Tileworld. Lect Notes Art Int 1209: $164-176$

Cody ML (1971) Finch flocks in the Mohave desert. Theor Popul Biol 2:142-158

Couzin ID, Krause J, James R, Ruxton GD, Franks NR (2002) Collective memory and spatial sorting in animal groups. J Theor Biol 218:1-11

Duostari MA, Sannomiya N (1995) A simulation study on schooling behaviour of fish in a water tank. Int J Syst Sci 26:2295-2306

Flierl G, Grunbaum D, Levin S, Olson D (1999) From individuals to aggregations: the interplay between behavior and physics. J Theor Biol 196:397-454

Hamilton WD (1971) Geometry for the selfish herd. J Theor Biol 31:295-311

Hiramatsu K, Shikasho S, Mori K (2000) Mathematical modeling of fish schooling of Japanese medaka using basic behavioral patterns. J Fac Agric Kyushu Univ 45:237-253

Huth A, Wissel C (1990) The movement of fish schools: a simulation model. In: Alt W, Hoffman G (eds) Biological motion. Springer-Verlag, Berlin, p 578-590

Huth A, Wissel C (1992) The simulation of the movement of fish schools. J Theor Biol 156:365-385

Inada Y, Kawachi K (2002) Order and flexibility in the motion of fish schools. J Theor Biol 214:371-387

Inagaki T, Sakamota W, Kuroki T (1976) Studies on the schooling behavior of fish. II. Mathematical modeling of schooling form depending on the intensity of mutual force between indivdiuals. Bull Jpn Soc Sci Fish 42:265-270

Krebs CJ (1989) Ecological methodology, 1st edn. Harper Collins, New York

Krebs JR, MacRoberts MH, Cullen JM (1972) Flocking and feeding in the great tit Parus major-and experimental study. Ibis 114:507-534

Lack D (1968) Ecological adaptations for breeding in birds, 1st edn. Chapman \& Hall, London

Matuda K, Sannomiya N (1980) Computer simulation of fish behavior in relation to fishing gear. I. Mathematical model of fish behavior. Bull Jpn Soc Sci Fish 46:689-697

Niwa H (1994) Self-organizing dynamic model of fish schooling. J Theor Biol 171:123-136

Niwa H (1996) Newtonian dynamical approach to fish schooling. J Theor Biol 181:47-63

Okubo A (1986) Dynamical aspects of animal grouping: swarms, schools, flocks and herds. Adv Biophys 22:1-94

Parrish JK (1992) Levels of diurnal predation on a school of flat-iron herring, Harengula thrissina. Environ Biol Fishes 24:257-263

Parrish JK, Edelstein-Keshet L (1999) Complexity, pattern, and evolutionary trade-offs in animal aggregation. Science 284:99-101

Parrish JK, Turchin P (1997) Individual decisions, traffic rules, and emergent pattern in schooling fish. In: Parrish JK, Hamner WM (eds) Animal groups in three dimensions, 1st edn. Cambridge University Press, Cambridge, p126-141

Parrish JK, Viscido SV, Grunbaum D (2002) Self-organized fish schools: an examination of emergent properties. Biol Bull 202:296-305

Partridge BL, Pitcher TJ (1980) The sensory basis of fish schools: relative roles of lateral line and vision. J Comp Physiol 135A:315-325

Pitcher TJ, Parrish JK (1993) Functions of shoaling behaviour in teleosts. In: Pitcher TJ (ed) Behaviour of teleost fishes, 2nd edn. Chapman \& Hall, New York, p 363-439

Pitcher TJ, Partridge BL, Wardle CS (1976) A blind fish can school. Science 194:963-965

Reuter H, Breckling B (1994) Self-organization of fish schools: an object-oriented model. Ecol Model 75:147-159

Ritz DA (1994) Social aggregation in pelagic invertebrates. Adv Mar Biol 30:156-216

Romey WL (1996) Individual diferences make a difference in the trajectories of simulated schools of fish. Ecol Model 92: $65-77$

Sannomiya N, Duostari MA (1996) A simulation study on autonomous decentralized mechanism in fish behaviour model. Int J Syst Sci 27:1001-1007

Shaw E (1978) Schooling fishes. Am Sci 66:166-175

Stocker S (1999) Models for tuna school formation. Math Biosci 156:167-190

Vabo R, Nottestad L (1997) An individual-based model of fish school reactions: predicting antipredator behaviour as observed in nature. Fish Oceanogr 6:155-171

Vine I (1971) Risk of visual detection and pursuit by a predator and the selective advantage of flocking behavior. J Theor Biol 30:405-422

Viscido SV, Wethey DS (2002) Quantitative analysis of fiddler crab flock movement: evidence for 'selfish herd' behaviour. Anim Behav 63:735-741

Warburton K, Lazarus J (1991) Tendency-distance models of social cohesion in animal groups. J Theor Biol 150:473-488

Watt PJ, Nottingham SF, Young S (1997) Toad tadpole aggregation behaviour: evidence for a predator avoidance function. Anim Behav 54:865-872

Wilson EO (1975) Sociobiology: the new synthesis, 1st edn. Harvard University Press, Cambridge, MA

Wu J, David JL (2002) A spatially explicit hierarchical approach to modeling complex ecological systems: theory and applications. Ecol Model 153:7-26

Submitted: April 16, 2003; Accepted: March 30, 2004

Proofs received from author(s): April 22, 2004 